\title{
Cardiac transplantation: The Stanford experience in the cyclosporine era
}

We analyzed our experience with 496 patients who underwent primary cardiac transplantation since the introduction of cyclosporine immunosuppression (Dec. 16, 1980, to Jan. 7, 1993). There were 388 male and 108 female patients. Mean recipient age was $40 \pm 16$ years (range 0.1 to 70 years, median 44 years). Recipient diagnoses included coronary disease in 188, idiopathic cardiomyopathy in 196, viral cardiomyopathy in 35, and congenital heart disease in 28 patients. Donor age was $25 \pm 10$ years (range 1 to 53 years, median 24 years). Graft ischemic time was $148 \pm 57$ minutes (range 38 to 495 minutes, median 149 minutes). Operative mortality (hospital death) rate was $7.9 \% \pm 1.3 \%(70 \%$ confidence intervals). Multivariate logistic regression analysis revealed that (higher) pulmonary vascular resistance and gender (female) were the only independent predictors of hospital death $(p<0.05)$. Actuarial survival estimates for all patients at 1, 5, and 10 years are $82 \% \pm 1.7 \%(83 \% \pm 1.8 \%$ adult, $77 \% \pm 5.2 \%$ pediatric), $61 \% \pm 2.5 \%(65 \% \pm 2.5 \%$ adult, $64 \% \pm 6.6 \%$ pediatric $)$, and $41 \% \pm 3.7 \%(40 \% \pm 4 \%$ adult, $54 \% \pm 8.6 \%$ pediatric), respectively. For 232 patients treated with triple-drug immunosuppression and induction with OKT3 since 1987, survival estimates at 1 and 5 years are $82 \% \pm 2.6 \%$ and $67 \% \pm 3.7 \%$, respectively. Causes of death for the entire group were rejection in $29(14 \%$ of deaths), infection in $69(34 \%)$, graft coronary disease in $36(18 \%)$, nonspecific graft failure in $6(3 \%)$, malignancy in $19(10 \%)$, stroke in $6(3 \%)$, pulmonary hypertension in $6(3 \%)$, and other causes in $30(15 \%)$ patients. Actuarial freedom from rejection at 3 months, 1 year, and 5 years was $21 \% \pm 1.9 \%, 14 \% \pm 1.7 \%$, and $7.2 \% \pm 1.5 \%$, respectively $( \pm 1$ standard error of the mean). Estimates of freedom from rejection-related death at 1,5 , and 10 years were $96 \% \pm 1 \%$, $93 \% \pm 1.4 \%$, and $93 \% \pm 1.4 \%$, respectively. Actuarial freedom from any infection at 3 months and at 1 and 5 years was $40 \% \pm 2.3 \%, 27 \% \pm 2.1 \%$, and $15 \% \pm 2.0 \%$ and from infection-related death, $95 \% \pm 1.0 \%, 93 \% \pm 1.2 \%$, and $85 \% \pm 1.9 \%$, respectively. Actuarial freedom from (angiographic or autopsy proved) graft coronary artery disease at $\mathbf{1 ,} 5$, and 10 years was $95 \% \pm 1.2 \%$,

George E. Sarris, MD, ${ }^{a}$ Kathleen A. Moore, BS, ${ }^{a}$ John S. Schroeder, MD, ${ }^{b}$

Sharon A. Hunt, MD, ${ }^{b}$ Michael B. Fowler, MD, ${ }^{b}$ Hannah B. Valantine, MD, ${ }^{b}$

Randall H. Vagelos, MD, ${ }^{b}$ Margaret E. Billingham, MD, ${ }^{c}$ Philip E. Oyer, MD, PhD, ${ }^{a}$

Edward B. Stinson, MD, ${ }^{a}$ Bruce A. Reitz, MD, and Norman E. Shumway, MD, PhD, ${ }^{a}$

Stanford, Calif.

From the Division of Cardiovascular Medicine, ${ }^{b}$ Department of Pathology, ${ }^{\mathrm{c}}$ and Department of Cardiothoracic Surgery, ${ }^{\mathrm{a}}$ Stanford University School of Medicine, Stanford, Calif.

Rẹad at the Nineteenth Annual Meeting of The Western Thoracic Surgical Association, Carlsbad, Calif., June 23-26, 1993.

\author{
Address for reprints: Norman E. Shumway, MD, PhD, Department of \\ Cardiothoracic Surgery, Falk Cardiovascular Research Center, \\ Stanford University School of Medicine, Stanford, CA 94305. \\ J ThoraC CARDIOvaSC Surg 1994;108:240-52 \\ Copyright $\odot 1994$ by Mosby-Year Book, Inc. \\ $0022-5223 / 94 \$ 3.00+0 \quad 12 / 6 / 55598$
}


$73 \% \pm 2.7 \%$, and $\mathbf{6 5} \% \pm 3.6 \%$ and from coronary disease-related death or retransplantation $98 \% \pm 0.7 \%, 84 \% \pm 2.2 \%$, and $66 \% \pm 4.3 \%$, respectively. Multivariate (Cox model) proportional hazard regression analysis revealed that (older) recipient age and race (nonwhite) were independent predictors of late (as opposed to hospital) death $(p<0.05)$. Immunosuppressive protocol was an independent predictor $(\boldsymbol{p}<\mathbf{0 . 0 5})$ of freedom from rejection (but not from late death or from rejectionrelated death). These data demonstrate satisfactory long-term results of cardiac transplantation for treatment of patients with end-stage heart disease. (J THORAC CardovasC SurG 1994;108:240-52)

\section{$\mathrm{T}_{\mathrm{h}}$} he introduction of cyclosporine in 1980 as the principal immunosuppressive agent in cardiac transplantation at Stanford ${ }^{1}$ has been associated with improved results ${ }^{2}$ and a dramatic increase in the number of cardiac transplants performed worldwide. Since that time, a substantial experience has accumulated at Stanford, where continued refinements in patient management, including immunosuppressive techniques, have been made. The purpose of this report is to review the overall long-term results of cardiac transplantation at our institution in the cyclosporine era and to identify risk factors (donor, recipient, or treatment-related) for transplant-related morbidity and mortality.

\section{Methods}

Patients. Between December 16, 1980, and January 7, 1993, 496 patients underwent primary orthotopic cardiac transplantation for end-stage heart disease at Stanford University Medical Center, and all received cyclosporine-based immunosuppression. Patients who underwent retransplantation $(n=34)$ were included in survival data analysis but were excluded (withdrawn alive) from further analysis of all other transplantrelated complications after the date of retransplantation. Selected patient characteristics are listed in Table I. Recipient diagnoses included coronary artery disease (CAD) in $188(38 \%)$, idiopathic cardiomyopathy in $196(40 \%)$, viral cardiomyopathy in $35(7 \%)$, congenital heart disease in $28(6 \%)$, postpartum cardiomyopathy in $11(2 \%)$, familial cardiomyopathy in $7(1 \%)$, and valvular disease in $12(2 \%)$ patients (Fig. 1). The series comprised 388 male and 108 female patients. Mean recipient age was $40 \pm 16$ years $( \pm 1$ standard deviation, $S D$; range 0.1 to 70 years, median 44 years). Donor age was $25 \pm 10$ years (range 1 to 53 years, median 24 years) (Fig. 2). Recipient and donor selection criteria have been previously described ${ }^{3,4}$ and have remained fairly constant over time; an exception to this is recipient age, with the original upper age limit of 50 years having been considerably extended for otherwise suitable potential recipients.

Operative techniques. Operative techniques have not changed significantly during the study period. Donor heart preservation has been with cold perfusion (with the Stanford hyperkalemic crystalloid cardioplegic solution) and topical cooling, followed by cold $\left(1^{\circ}\right.$ to $\left.4^{\circ} \mathrm{C}\right)$ storage. The technique of graft implantation has not changed since its original description. ${ }^{5}$ Topical cooling of the donor heart is maintained during implantation by continuous cold saline irrigation of the pericardial well. In addition, after completion of the left atrial anastomosis, a continuous infusion of cold saline through the left atrial appendage provides additional endocardial cooling and assists with evacuation of air from the left side of the heart. Graft ischemic time was $148 \pm 57$ minutes (range 38 to 495 minutes, median 149 minutes).

Immunosuppression. Although all patients received cyclosporine, the overall immunosuppressive protocol has changed over time, as accumulating experience with this agent prompted changes in clinical management. High cyclosporine doses were used initially, but these were subsequently reduced in an effort to avoid nephrotoxicity. Tapering schedules of steroids have been used, and azathioprine has been adjusted according to the white blood cell count. Polyclonal rabbit antithymocyte globulin (Stanford) and, subsequently, horse antithymocyte globulin (ATGAM, Upjohn Co., Kalamazoo, Mich.) were used early in our experience. Starting in 1987 and continuing to date, induction therapy with OKT3 has been used. A recent cohort of patients was randomized to induction with either OKT3 or (group $\mathbf{J}$ ) an anti-CD4 chimeric monoclonal antibody (CMT412, referred to as OKT4 in this study, Centocor Inc., Malvern, $\mathrm{Pa}$.). The results of this prospective, randomized study are now being analyzed and will be reported separately. The components of the immunosuppressive protocols used (labeled groups C, D, E, F, G, H, I, and J) are summarized in Table II.

The current protocol (group I) includes cyclosporine initiated after the operation and titrated to maintain a trough serum concentration (as measured by fluorometric polarization immunoassay, Abbott Laboratories, Abbott Park, Chicago, Ill.) of 150 to $250 \mathrm{ng} / \mathrm{ml}$ in the first few weeks after transplantation and 100 to $150 \mathrm{ng} / \mathrm{ml}$ thereafter. Azathioprine is administered intravenously at $4 \mathrm{mg} / \mathrm{kg}$ before the operation and subsequently maintained at approximately $2 \mathrm{mg} / \mathrm{kg}$ per day, adjusted to maintain the white blood cell count greater than 5000 . Methylprednisolone is administered during the operation $(500 \mathrm{mg}$ at the conclusion of cardiopulmonary bypass) and after the operation ( $125 \mathrm{mg}$ every 8 hours for three doses). Prednisone is then started at a daily oral dose of $0.6 \mathrm{mg} / \mathrm{kg}$ and gradually tapered over the ensuing 2 months to $0.2 \mathrm{mg} / \mathrm{kg}$ per day. Murine monoclonal antibody (OKT3) is given intravenously ( $5 \mathrm{mg} /$ day) daily for 14 days.

Rejection was detected on routine endomyocardial biops $y^{6}$ or suspected on clinical grounds and confirmed by biopsy in most instances. Moderate or severe rejection has been treated with intravenous methylprednisolone (1 gm/day for 3 days), followed by a 2-week prednisone tapering schedule. Rejection persistent despite two courses of steroid therapy has been managed with antithymocyte globulin or OKT3. Total lymphoid irradi- 


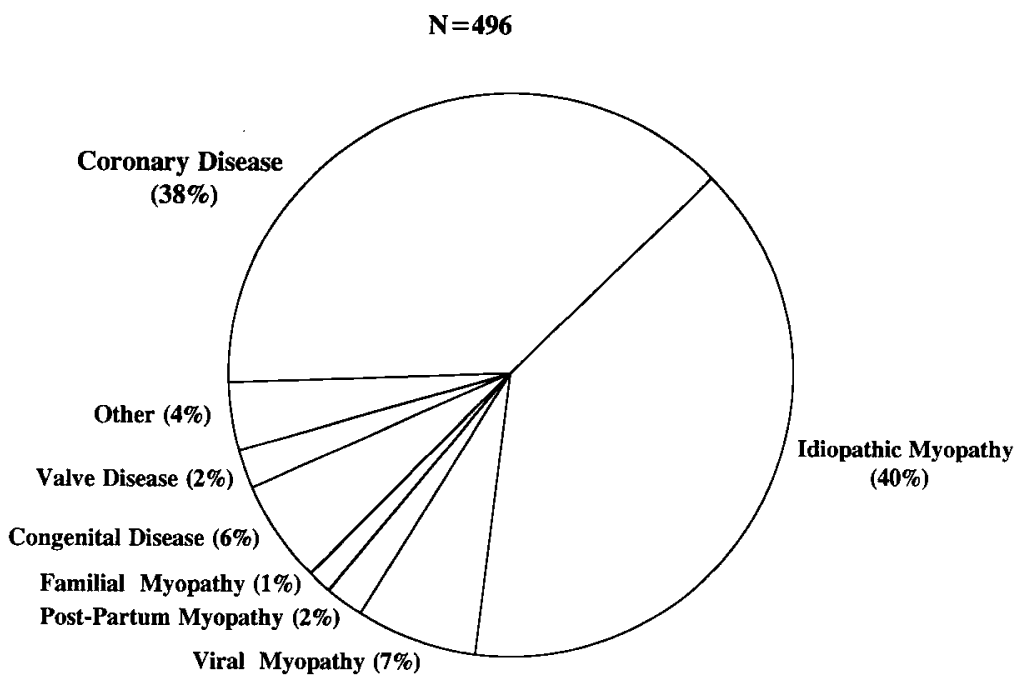

Fig. 1. Distribution of recipient diagnoses.

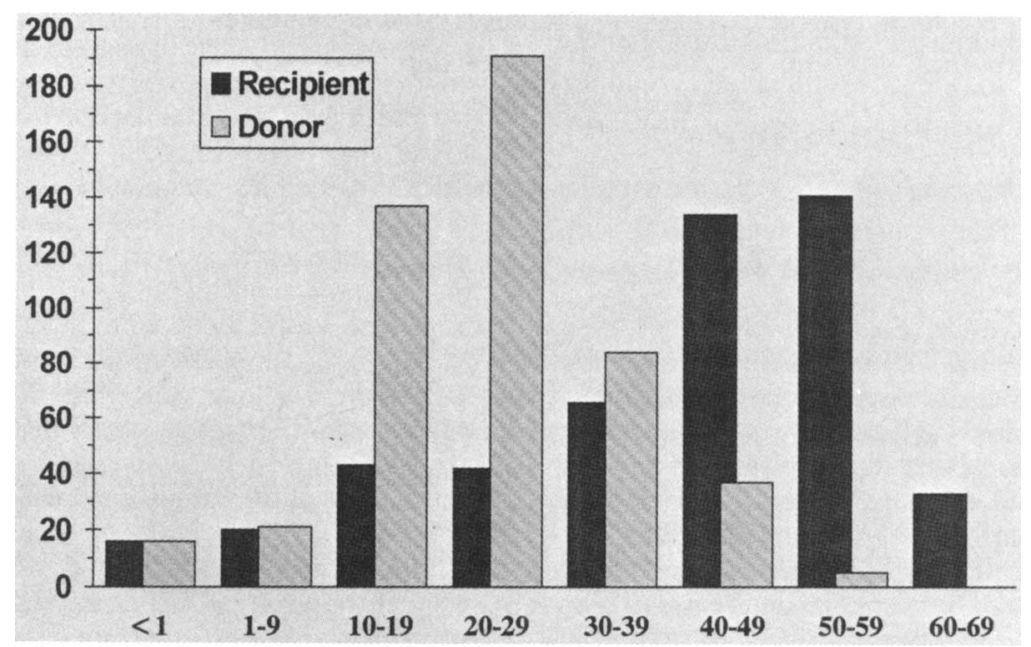

Fig. 2. Age distribution of the recipient and donor patient populations.

ation or methotrexate has been used as adjunctive therapy for more recalcitrant rejection.

Follow-up. Discharged patients have been observed closely either locally or in close communication with the primary care physician and have undergone yearly detailed evaluation that has included, in most instances, cardiac catheterization, endomyocardial biopsy, and coronary angiography. Mean follow-up time was $4.3 \pm 3.3$ (SD) years. Morbid and fatal transplantrelated events were categorized as rejection (diagnosed on the basis of endomyocardial biopsy or autopsy), infection, and graft CAD (on the basis of angiography and/or autopsy). Cause of death was ascertained carefully in all patients, including an autopsy in most $(>90 \%)$ cases. Data have been prospectively collected and entered into the Stanford Transplant Database, which was also used to perform statistical analyses.

Statistical analysis. For continuous data, variability is reflected by $\pm 1 \mathrm{SD}$. Variability of important ratios is expressed as $\pm 70 \%$ confidence limits. Comparisons between continuous variables were made with an unpaired two-tailed $t$ test, and discrete variables were compared with the continuity-adjusted $\chi^{2}$ test. Actuarial life-table data were calculated by the CutlerEderer method, ${ }^{7}$ which also generated the cumulative hazard functions. The actuarial estimates were used to describe the time-related event-free rates from death and other transplantrelated complications, and variability of these estimates is indicated by \pm 1 standard error of the mean. Comparisons between actuarial curves were made by the Lee-Desu method. ${ }^{8}$

Multivariate logistic regression analysis was used to assess which recipient or donor-related variables (preoperative or treatment-related) were important predictors of operative mortality (defined as death within 30 days of operation or death in the hospital before discharge after transplantation). Multivariate (Cox model) proportional hazard regression analysis was used to assess which such variables were significant, independent predictors of late transplant-related complications (SPSS statistical software, SPSS Inc., Chicago, Ill.). ${ }^{8}$ The following vari- 
Table II. Summary of immunosuppressive protocols used

\begin{tabular}{crcccc}
\hline Group & No & $C y$ & Aza & Pred & Antibody \\
\hline C & 26 & + & - & + & RATG \\
D & 78 & $+\downarrow$ & - & + & - \\
E & 40 & $+\downarrow \downarrow$ & + & + & ATGAM \\
F & 22 & $+\downarrow \downarrow$ & + & - & ATGAM \\
G & 34 & $+\downarrow \downarrow$ & + & $+\downarrow$ & ATGAM \\
H & 55 & $+\downarrow \downarrow \downarrow$ & + & $+\downarrow$ & - \\
I & 232 & $+\downarrow \downarrow \downarrow$ & + & $+\downarrow$ & OKT3 \\
J & 9 & $+\downarrow \downarrow \downarrow$ & + & $+\downarrow$ & OKT4
\end{tabular}

Cy, Cyclosporine. Initially (group C), high-dose ( $18 \mathrm{mg} / \mathrm{kg}$ per day) cyclosporine was used. In later groups, cyclosporine doses were gradually reduced to current levels, as denoted by the downward arrows. Aza, Azathioprine; Pred, prednisone (downward arrow indicates reduced dosage to current level); RATG, rabbit antithymocyte globulin (Stanford); $A T G A M$, horse antithymocyte globulin (Upjohn Co, Kalamazoo, Mich.); OKT4, monoclonal anti-CD4 antibody (Centocor Inc., Malvern, $\mathrm{Pa}$.)

tion in 3 ( $8 \%$ of deaths), infection in $19(49 \%)$, nonspecific graft failure in $4(10 \%)$, pulmonary hypertension in $6(15 \%)$, pulmonary embolus in $1(3 \%)$, stroke in $2(5 \%)$, and other causes in $4(10 \%)$ patients (Fig. 3, $A$ ).

Multivariate logistic regression analysis revealed that preoperative (higher) PVR and gender (female) were the only independent predictors of hospital death $(p<0.05)$. A continuous positive relationship between preoperative PVR and risk of hospital death was demonstrated. To further illustrate this point, we compared mortality rates for patients with PVR greater and less than 5 Wood units. This value of PVR was selected arbitrarily for comparison purposes because it represents the mean \pm 1 SD of the PVR values for the entire study population and because a PVR of 5 to 6 units has been clinically considered to portend higher risk at our institution. Operative mortality for patients with PVR greater than 5 Wood units $(n=32)$ was $28 \% \pm 8 \%$ versus $8 \% \pm 2 \%$ for those with PVR less than 5 Wood units $(p=0.04)$.

Operative mortality was $16 \% \pm 3 \%$ for women versus $6 \% \pm 1 \%$ for men $(p=0.02)$. This difference could not be attributed to differences in recipient PVR, donor/recipient weight ratios, or any other variable. Donor/recipient weight ratio $(1.12 \pm 0.44 \mathrm{~kg}[$ mean $\pm 1 \mathrm{SD}])$ and preoperative status (need for inotropic or mechanical support) were not independent risk factors for operative death.

Recipient age (pediatric) and donor gender (female) were associated with increased risk of hospital death in the univariate screening process, but these variables dropped out from the multiple regression equation when PVR was entered in the analysis. Operative mortality for patients under 18 years of age $(n=67)$ was $15 \% \pm 5 \%$, compared with $7 \% \pm 1.3 \%$ for patients over age 18 years $(n=429$, $p=0.02$ ). 


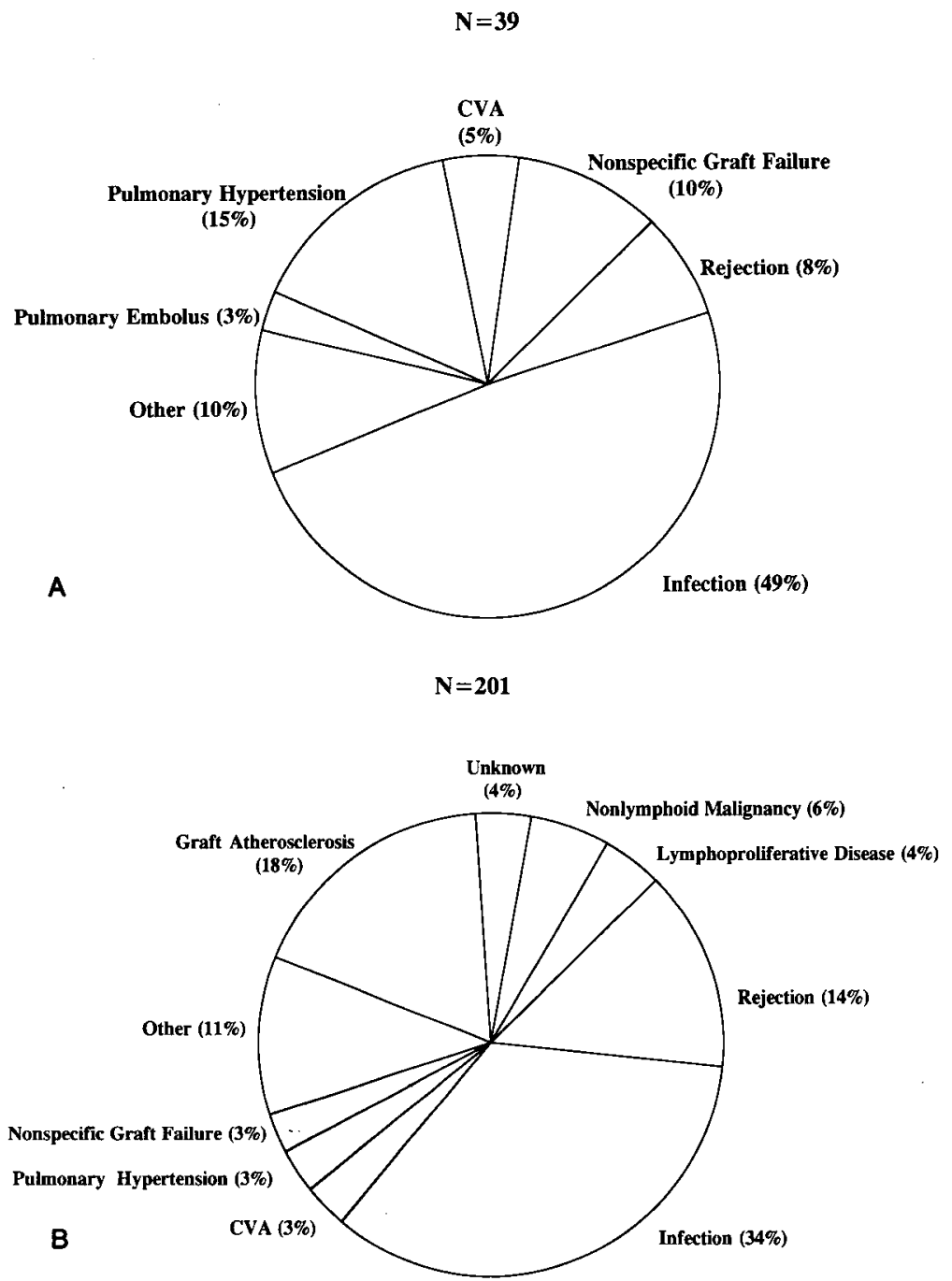

Fig. 3. Causes of operative (A) and overall deaths (B).

Long-term survival. Of the 496 patients in this series, 295 are currently alive, 286 with the original graft and 9 after retransplantation (a total of 34 patients have undergone retransplantation). Most patients $(232,81 \%)$ are currently fully rehabilitated, and most $(224,78 \%)$ are in New York Heart Association functional class I.

A total of 201 deaths occurred (39 early, 162 late). Actuarial survival estimates for all patients at 1,5 , and 10 years are $82 \% \pm 1.7 \%, 61 \% \pm 2.5 \%$, and $41 \% \pm 3.7 \%$, respectively (Fig. 4). Causes of death (both early and late) for the entire group were rejection in 29 (14\% of all deaths), infection in $69(34 \%)$, graft CAD in $36(18 \%)$, nonspecific graft failure in $6(3 \%)$, lymphoproliferative malignancy in $8(4 \%)$, nonlymphoid malignancy in 11 $(6 \%)$, stroke in $6(3 \%)$, pulmonary hypertension in $6(3 \%)$, and other causes in $30(15 \%)$ patients (Fig. 3, B).
Multivariate (Cox model) proportional hazard regression analysis revealed that (older) recipient age and race (nonwhite) were independent predictors of late (as opposed to hospital) death $(p<0.05)$. Transplant year and immunosuppressive protocol did not emerge as independent predictors of late death (univariate $p$ values of 0.06 and 0.43 , respectively).

Comparisons of actuarial survival curves according to decade of age at transplantation suggested that pediatric patients (aged $<18$ ) have lower early but improved longterm survival: actuarial survival estimates at 1,5 , and 10 years are $83 \% \pm 1.8 \%, 65 \% \pm 2.5 \%$, and $40 \% \pm 4 \%$ for adults and $77 \% \pm 5.2 \%, 64 \% \pm 6.6 \%, 54 \% \pm 8.6 \%$ for pediatric patients, respectively. However, the differences between these curves were not statistically significant.

Survival estimates at 1,5 , and 10 years were 


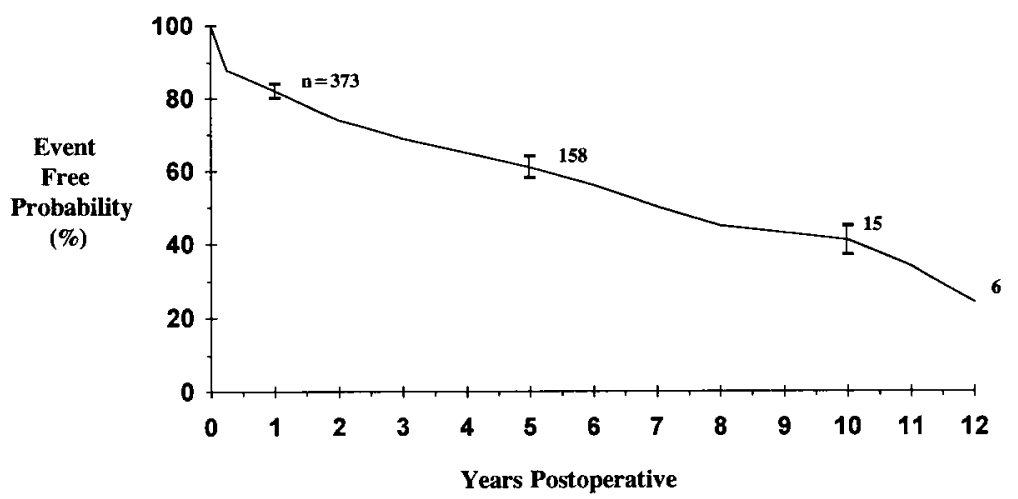

Fig. 4. Actuarial survival for the entire patient population. Error bars in this and all figures represent \pm 1 standard error of the mean.

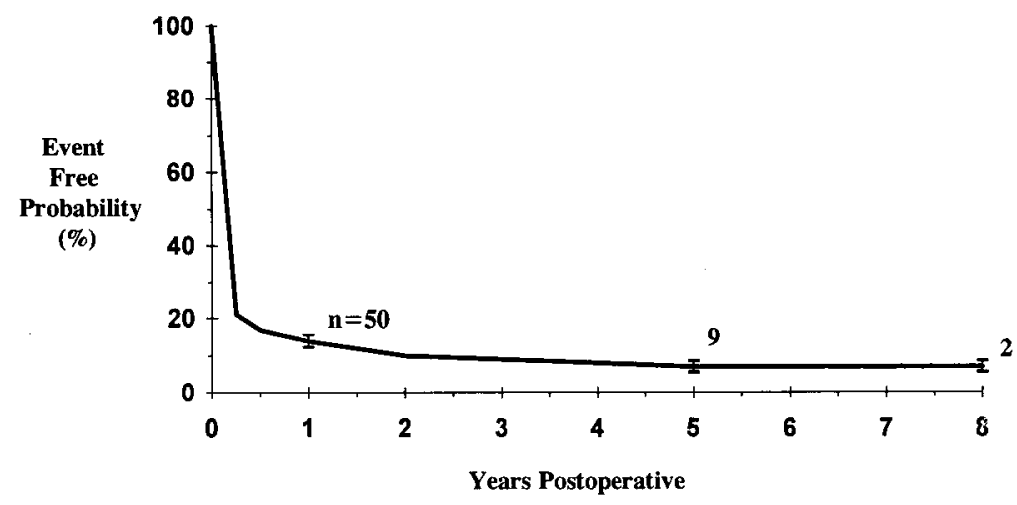

Fig. 5. Actuarial freedom from rejection for the entire group of patients.

$81 \% \pm 1.9 \%, 62 \% \pm 2.6 \%$, and $42 \% \pm 4.0 \%$ for white $(n=417) \quad$ and $\quad 87 \% \pm 4.0 \%, \quad 54 \% \pm 7.2 \%, \quad$ and $29 \% \pm 10.8 \%$ for nonwhite $(n=77)$ patients, respectively, but this difference was not statistically significant. Furthermore, no significant difference was detected between these two groups of patients in terms of causes of death.

Although transplant year and immunosuppressive protocol did not emerge as independent predictors of longterm outcome, survival estimates of the 232 patients treated with the current regimen of triple-drug immunosuppression and induction with OKT3 since 1987 were calculated; at 1 and 5 years these estimates are $82 \% \pm 2.6 \%$ and $67 \% \pm 3.8 \%$, respectively $\left(p=\mathrm{NS}^{*}\right.$ compared with all other patients, $82 \% \pm 2.4 \%$ and $63 \% \pm 3 \%$, respectively).

Rejection. Multivariate (Cox model) proportional hazard regression analysis revealed that only immuno-

${ }^{*} \mathrm{NS}=$ Not significant suppressive protocol, specifically, group I (the current regimen of triple-drug immunosuppression and induction OKT3) was an independent predictor $(p<0.05)$ of freedom from rejection (but not from rejection-related death). Group F (cyclosporine and azathioprine only) was associated with an increased risk of rejection $(p<0.05)$. Importantly, although earlier transplant year was associated with an increased risk of rejection in the univariate screening process $(p<0.05)$, transplant year did not emerge as an independent predictor of freedom from rejection in the multivariate analysis. Furthermore, no variable was identified as an independent predictor of freedom from rejection-related death.

Actuarial freedom from rejection at 3 months, 1 year, and 5 years was $21 \% \pm 1.9 \%, 14 \% \pm 1.7 \%$, and $7 \% \pm 1.5 \%$, respectively, for the entire group of patients (Fig. 5). For immunosuppressive group I, these estimates were $25 \% \pm 3.0 \%, 16 \% \pm 2.6 \%$, and $8.2 \% \pm 2.4 \%$, respectively ( $p<0.01$ versus all other patients). A significant increase in the time to first rejection episode was 


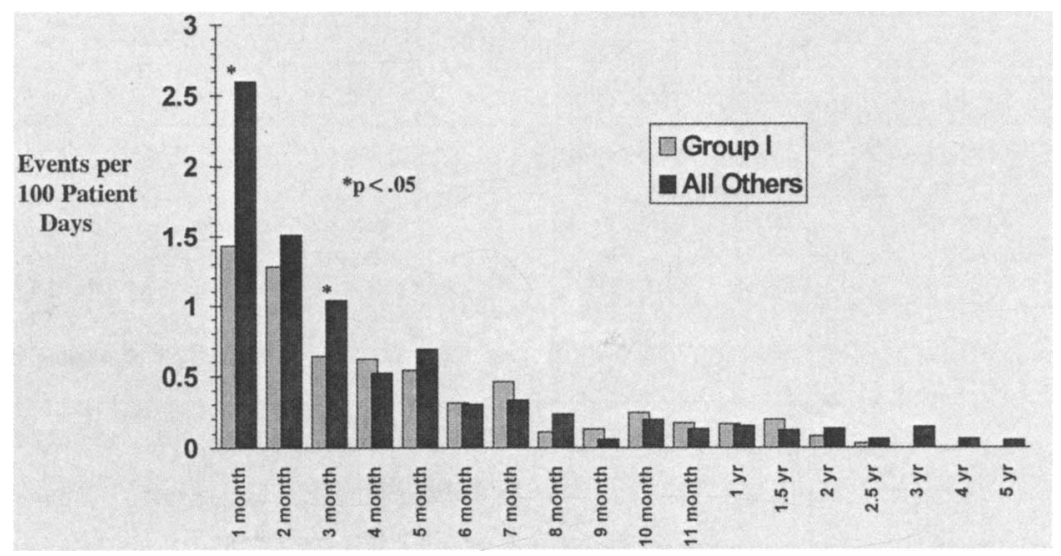

Fig. 6. Linearized rejection rates for current protocol (group I) and all other patients.

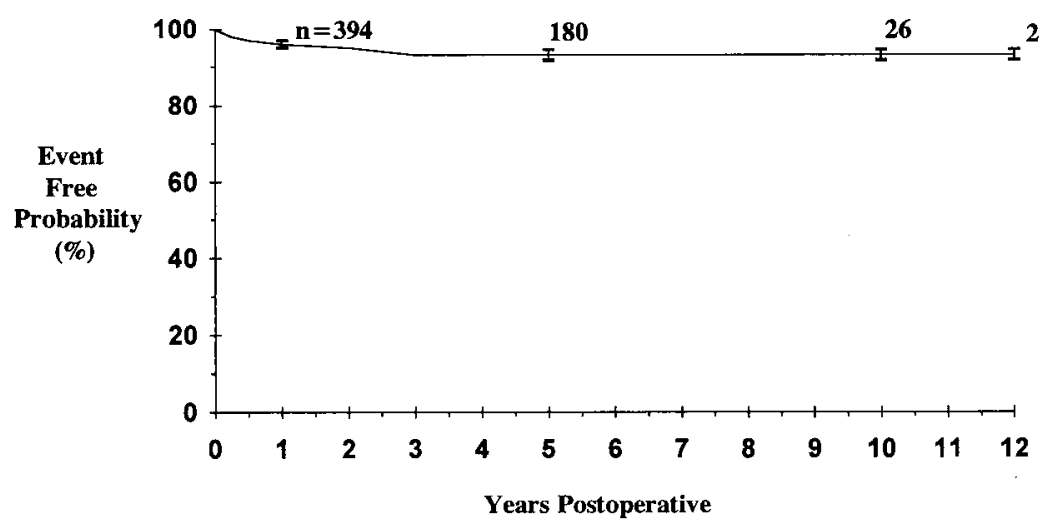

Fig. 7. Actuarial freedom from rejection-related death for the entire group of patients.

noted in group I versus all other groups $(53 \pm 70$ versus $30 \pm 41$ days). Because most patients had at least one rejection episode within the first 6 months after transplantation, linearized rejection rates were also calculated: expressed as number of rejection episodes per 100 patientdays, the linearized rejection rates for the entire group of patients during the first, third, and sixth months after transplantation were $2.04 \pm 0.12,0.85 \pm 0.08$, and $0.30 \pm 0.05$, respectively. For immunosuppressive group I, these figures were $1.43 \pm 0.15(p<0.00002$ versus all other patients), $0.64 \pm 0.1(p<0.02)$, and $0.31 \pm 0.07$ $(p=$ NS), respectively (Fig. 6).

Estimates of freedom from rejection-related death at 1 , 5 , and 10 years were $96 \% \pm 1 \%, 93 \% \pm 1.4 \%$, and $93 \% \pm 1.4 \%$, respectively (Fig. 7).

Infection. Actuarial freedom from any infection at 3 months, 1 year, and 5 years was $40 \% \pm 2.3 \%$, $27 \% \pm 2.1 \%$, and $15 \% \pm 2.0 \%$ and from infection-related death $95 \% \pm 1.0 \%, 93 \% \pm 1.2 \%$, and $85 \% \pm 1.9 \%$, respectively (Fig. 8, $A$ and $B$ ). The risk of infection and infection-related death is highest early after transplantation, then rapidly declines, but persists at a low rate thereafter. Actuarial freedom from infection with the major classes of pathogens is shown in Fig. 8, $C$.

Multivariate analysis revealed that (earlier) transplant year was the only independent predictor of increased risk of infection $(p<0.05)$. The reduced incidence of (early) infection in the more contemporary cohort of patients (1987 to 1993) compared with the earlier cohort (1980 to $1986)$ is also evident $(p<0.01)$ (Fig. 8, $D$ ).

Graft coronary disease. Actuarial freedom from (angiographic or autopsy-proved) graft CAD at 1, 5, and 10 years was $95 \% \pm 1.2 \%, 73 \% \pm 2.7 \%$, and $65 \% \pm 3.6 \%$, from CAD-related death $99 \% \pm 0.3 \%$, $90 \% \pm 1.8 \%$, and $81 \% \pm 3.5 \%$, and from CAD-related death or retransplantation $98 \% \pm 0.7 \%, 84 \% \pm 2.2 \%$, and $66 \% \pm 4.3 \%$, respectively (Fig. 9, $A, B$, and $C$ ).

Multivariate analysis demonstrated the following factors to be associated with an increased risk of graft CAD: older donor age, older recipient age, earlier transplant 

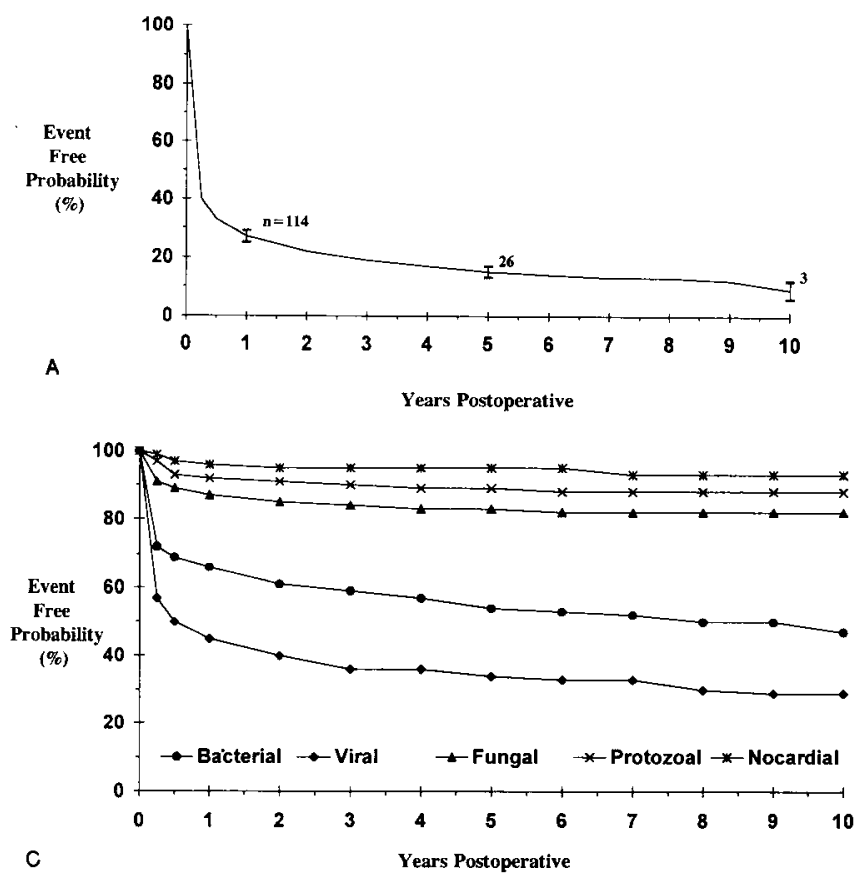
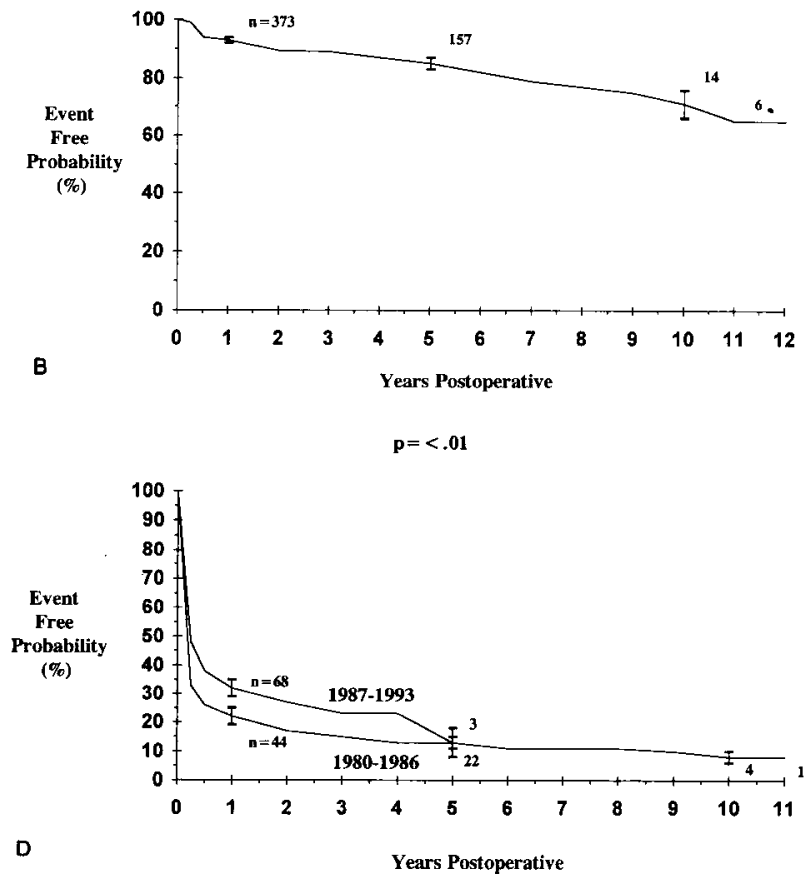

Fig. 8. Actuarial freedom from infection (A) and from infection-related death (B) for all patients. C, Actuarial freedom from infection with the major classes of pathogens. D, Actuarial freedom from infection for patients operated on before and after 1987.
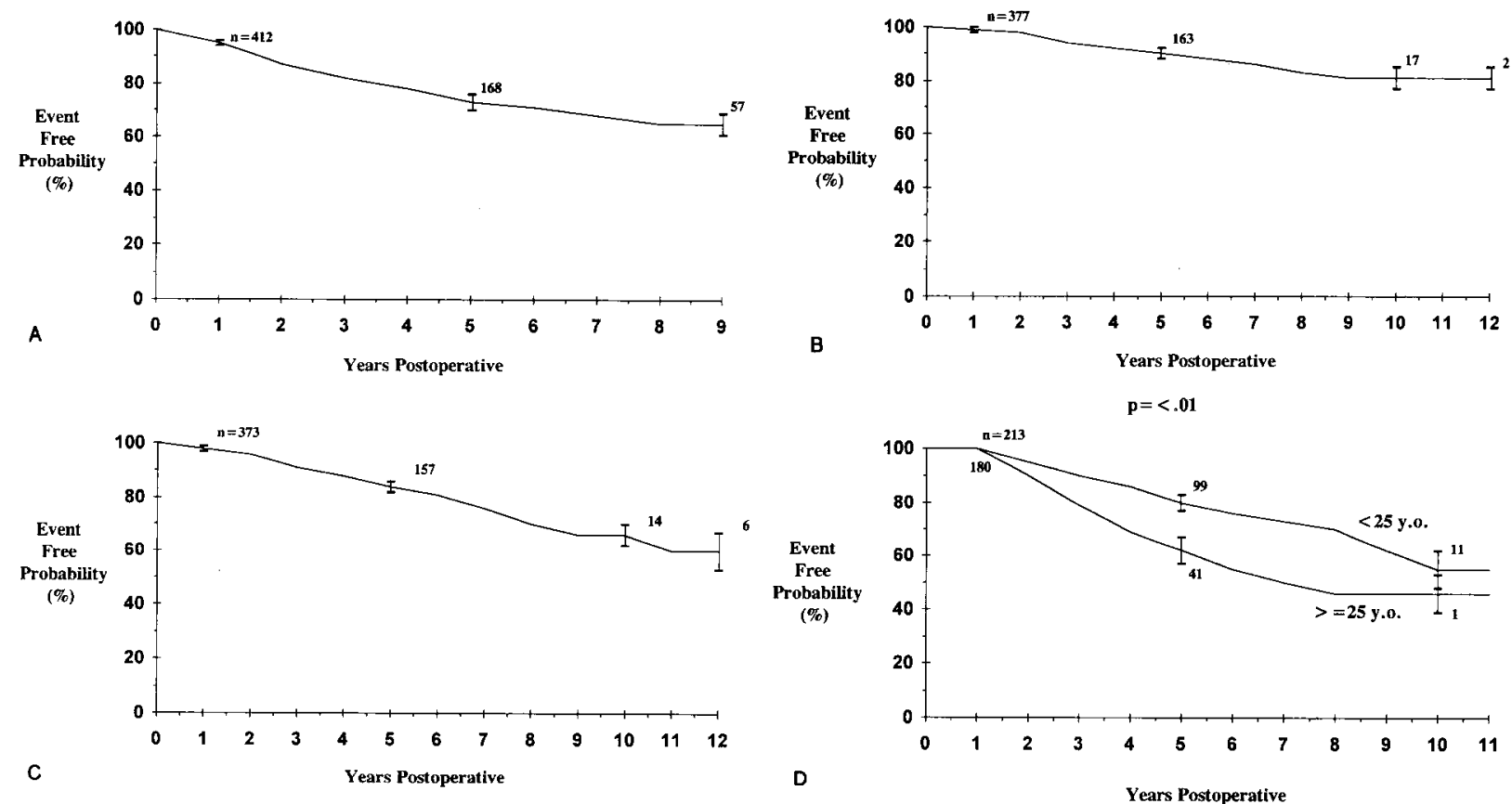

Fig. 9. Actuarial freedom from graft CAD (A) from CAD-related death (B), and from CAD-related death or retransplantation $(\mathbf{C})$. D, Actuarial freedom from graft $\mathrm{CAD}$ according to donor age.

year, and immunosuppressive protocol (group $\mathrm{H}$ ) $(p<0.05)$.

By actuarial analysis, only $62 \% \pm 5 \%$ of patients with donor age greater than 25 years were free of CAD at 5 years, compared with $80 \% \pm 3 \%$ of those with donor age less than 25 years (Fig. 9, D) $(p<0.01)$. A similar trend regarding recipient age did not achieve statistical significance: $69 \% \pm 0.4 \%$ of recipients aged 41 years or older 


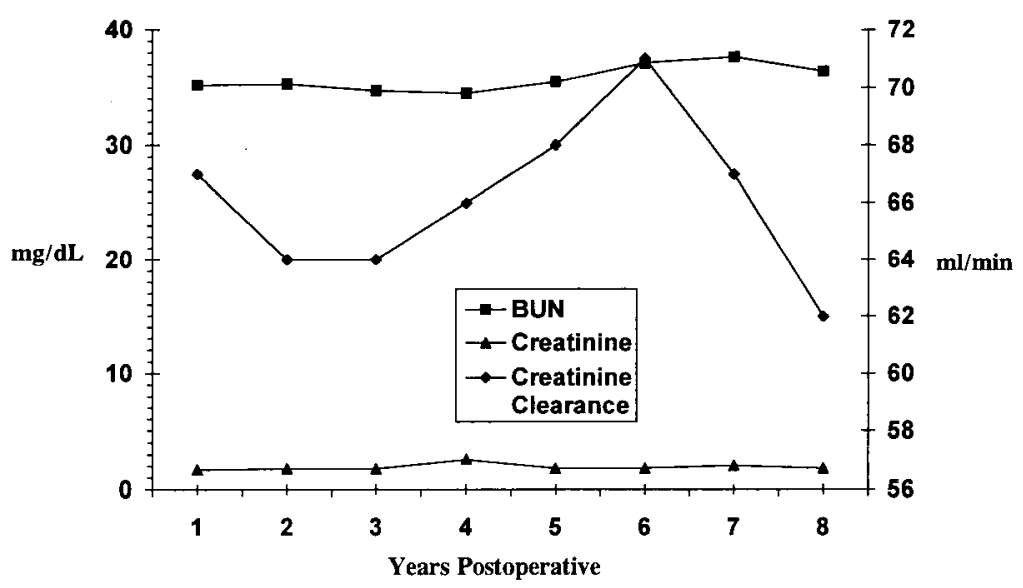

Fig. 10. Renal function parameters remained relatively stable after an initial decline early after the operation. $B U N$, Blood urea nitrogen.

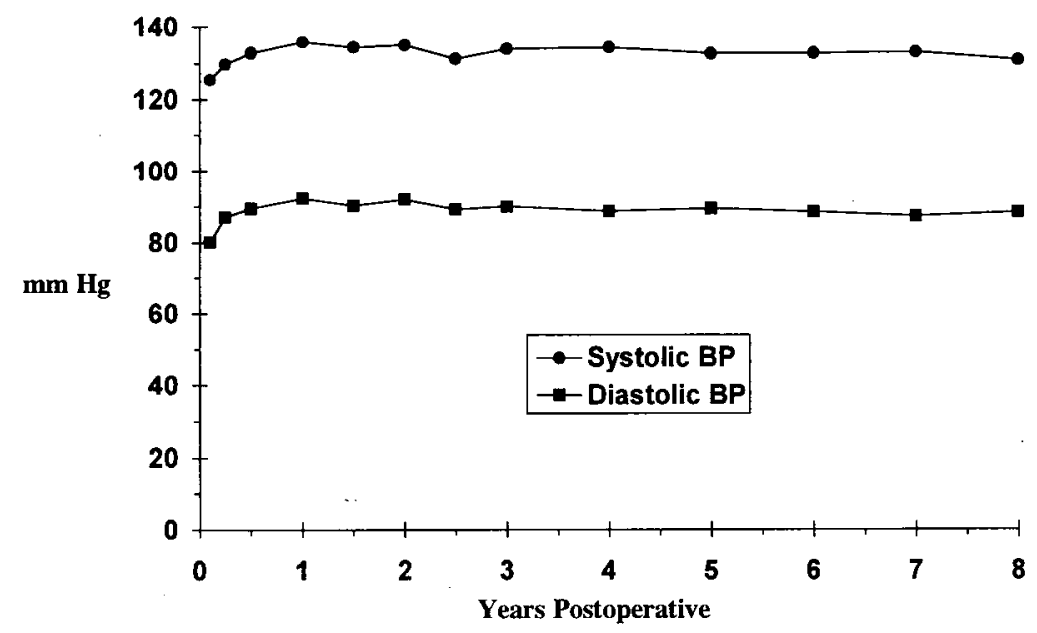

Fig. 11. Blood pressure $(B P)$ rises early after transplantation but remains stable thereafter.

were free of CAD at 5 years, compared with $78 \% \pm 4 \%$ of those younger than 41 years at the time of transplantation $(p=0.23)$. By actuarial analysis, the observed differences in the rates of CAD according to transplant year (decreased prevalence in the more contemporary group of patients) did not achieve statistical significance. Finally, immunosuppressive group $\mathrm{H}$ (triple-drug therapy) had significantly higher $(p<0.02)$ rates of graft CAD than all other groups, but the rates of graft CAD were not significantly better for the current protocol (group I) receiving triple-drug therapy plus induction OKT3.

Renal function. Renal function parameters (blood urea nitrogen, serum creatinine, and creatinine clearance) trends are illustrated in Fig. 10 and show possible mild deterioration early after the operation, but stability in renal function over the long term. Average blood pressure data, shown in Fig. 11, demonstrate good long-term control of hypertension. Immunosuppressive medication doses are summarized in Fig. 12. The data reflect lower cyclosporine doses used in our most recent protocols, as well as the policy of allowing lower cyclosporine levels after the first few postoperative weeks. Azathioprine and steroid regimens have remained unchanged.

\section{Discussion}

Operative mortality. The overall operative mortality rate $(7.9 \% \pm 1.3 \%)$, defined as death within 30 days of transplantation or death in the hospital before discharge, has not changed appreciably over time and compares favorably with the less stringent 30-day mortality data ( $9 \%$ to $10 \%$ ) reported by the Registry of the International Society for Heart and Lung transplantation (ISHLT). ${ }^{9}$ 


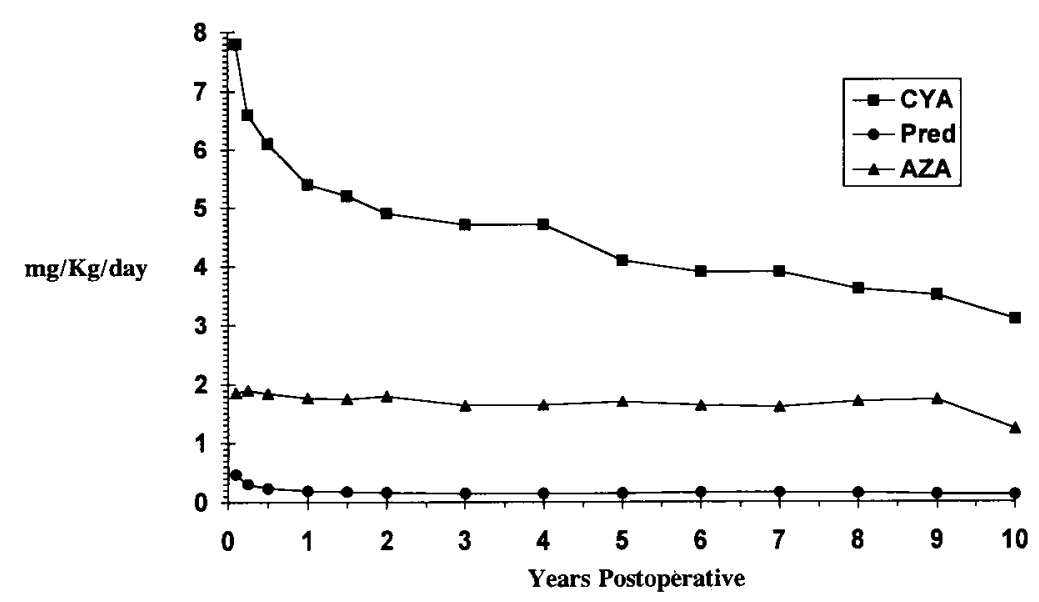

Fig. 12. Doses over time of the three primary immunosuppressive agents used. $C Y A$, Cyclosporine; Pred, prednisone; $A Z A$, azathioprine.

Operative mortality was clearly related to recipient age, with a significantly higher mortality for the pediatric population (age less than 18 years). Most deaths were due to infectious complications, the remaining being attributed mostly to rejection, nonspecific graft failure, and pulmonary hypertension. Of note, multivariate analysis revealed that pulmonary hypertension (and not recipient age) has a continuous positive relationship with and is an independent predictor of early death after transplantation. Patients with PVR in excess of 5 Wood units ( $>1$ SD higher than the group mean) had a significantly higher operative mortality, despite the fact that, in most cases, patients with elevated PVR were accepted as transplant candidates only after pharmacologic intervention (sodium nitroprusside and oxygen with or without inotropic support) demonstrated that pulmonary hypertension was not fixed. These results confirm previous reports from this and other institutions that elevation of PVR may cause right-sided heart failure in the early postoperative period, is a recognized risk factor for operative mortality after heart transplantation in both adults and children, and, when excessive or fixed, should represent an absolute contraindication to orthotopic transplantation..$^{10-12}$

Long-term survival. Actuarial survival estimates at 1 and 5 years remain essentially unchanged compared with those reported previously regarding our first 310 patients of the cyclosporine era. ${ }^{2}$ No difference in survivals was observed for the most recent cohort of patients (since 1987) receiving triple-drug immunosuppression and induction OKT3. Actuarial survival results for up to 10 years after operation are reported and compare favorably with worldwide data of the Registry of the International Society for Heart and Lung Transplantation, which indi- cate 1 -, 5-, and 10-year survival estimates of $78.29 \%$, $66.79 \%$, and $52.48 \%$, respectively. ${ }^{9}$

Multivariate analysis confirmed that (older) age at transplantation is a significant determinant of (reduced) long-term survival. Of note, pediatric patients (who had significantly higher early mortality, as detailed earlier) have comparable long-term survival with all other patients. On the other extreme of the age spectrum, carefully selected older patients (age $>60$ years) have recently been accepted as transplant candidates. Their operative mortality rate and medium-term survival have also been comparable with that of the entire group of patients. Although the 5-year actuarial survival of this older subgroup of patients is lower than the corresponding figure for middle-aged patients, this difference was not statistically significant. These results are similar to those reported from other centers. ${ }^{13,14}$

Rejection. Several immunosuppressive protocols were used during the study period, as summarized in Table II. Rejection occurred within the first 3 months after transplantation in most patients, with only $21 \% \pm 1.2 \%$ of patients remaining rejection-free at that time. The risk of rejection peaked during the first 3 months, and the hazard function remained relatively constant at a low level of risk after the first year. This pattern of rejection risk has been noted in previous reports from this ${ }^{2}$ and other institutions. ${ }^{15}$ Although earlier transplant year was associated with an increased risk of rejection, multivariate analysis demonstrated that only immunosuppressive protocol was an independent determinant of rejection, with group $\mathrm{H}$ being associated with increased and group I associated with reduced risk of rejection. The benefit in group I was limited to delaying the time to first rejection and to 
reducing early rejection rates. These findings confirm our prior experience with induction OKT $3,{ }^{16}$ as well as that of groups from Utah ${ }^{17}$ and the University of Alabama. ${ }^{18}$ The more recent report ${ }^{15}$ from the University of Alabama of increased risk of primary or repeated rejection with induction OKT3 represents an unusual experience; as the authors speculated, it may be paradoxically attributable to increased salvage from rejection-related death of patients with high risk of rejection, which, in their analysis, was a strong predisposing factor for recurrent rejection. Other risk factors for rejection identified in the Alabama series (younger recipient age, female recipient or donor gender, and number of HLA mismatches) were not significant determinants of rejection in our experience. Specifically, although $\mathrm{we}^{19}$ have previously reported a trend toward a higher number of deaths from infection and rejection in transplant recipients with three or four HLA (locus A or B) mismatches, the number of HLA mismatches was not an independent predictor of rejection in this analysis of our larger experience.

It should be clearly stated, however, that although our current immunosuppressive protocol (group I) was associated with increased freedom from rejection and reduced linearized rejection rates early after transplantation, these benefits did not persist beyond 3 months and did not translate into any demonstrable benefit in terms of death from rejection, infection, $\mathrm{CAD}$, and, most important, in overall survival.

Infection. Infection remains a major complication and cause of death for these immunosuppressed patients, both early and late after operation. The risk of infection and infection-related death is highest in the perioperative period and then rapidly declines over the first year after transplantation, but lower levels of risk persist throughout the postoperative follow-up period. Regarding risk factors for infection, only earlier operative year but not immunosuppressive protocol was identified as an independent predictor of infection. This suggests that the increased effectiveness of our current immunosuppressive protocol of triple-drug therapy and induction OKT3 in terms of ameliorating early rejection, as noted earlier, was not obtained at the cost of increased susceptibility to infection.

Graft CAD. Accelerated CAD in the transplanted heart remains a major complication, as previously reported from this ${ }^{20-27}$ and other ${ }^{28}$ centers, limiting long-term survival. It has previously been reported that the prevalence of this disease has not been reduced with the introduction of antiplatelet agents or cyclosporine. ${ }^{22}$ Older donor age and elevated plasma triglycerides have been previously identified as predisposing factors for graft $\mathrm{CAD},{ }^{21}$ and an association with $\mathrm{CMV}$ infection ${ }^{25}$ has also been noted. Number of rejection episodes, number of
HLA mismatches, and dosage of steroids have not been identified as risk factors for CAD. ${ }^{21}$ In the present retrospective study, multivariate analysis confirmed that older donor age was associated with increased risk of graft CAD and also revealed three additional risk factors: older recipient age, earlier transplant year, and immunosuppressive protocol (group H: triple-drug therapy without OKT3). Conversely, induction OKT3 did not ameliorate the prevalence of graft CAD. Of note, HLA matching, identical versus compatible blood type, recipient diagnosis, and donor or recipient CMV status were not found to be independent predictors of graft CAD, but the effect of documented postoperative CMV disease (infection) was not separately analyzed in this study. Furthermore, treatment with diltiazem did not emerge as an independent factor predicting freedom from CAD in this combined retrospective analysis, but it has been clearly shown to inhibit the development of CAD in the treatment group $(n=52)$ of a prospective, randomized trial of a recent (1986 to 1989) cohort of 106 cardiac transplant recipients. ${ }^{27}$

Given the trend to accept older patients as candidates for heart transplantation and the increasing use of older donors in response to the increasing shortage of organ donors, graft CAD is likely to become an even greater threat to long-term survival of cardiac transplant recipients. Its eventual control may depend on the development of either reliable protocols to achieve tolerance or methods to achieve specific inhibition of proliferation of cellular elements within the coronary arterial wall.

\section{Conclusion}

These data demonstrate satisfactory long-term results of orthotopic cardiac transplantation for treatment of patients with end-stage heart disease, who are known to have high mortality rates when treated medically. Nonetheless, despite recent advances, major barriers remain that prevent the realization of cardiac transplantation's full potential for the treatment of end-stage heart disease: Acute rejection remains incompletely controlled; graft CAD (believed to be a manifestation of chronic rejection) remains a major long-term complication; and current immunosuppressive management still leaves patients permanently vulnerable to the risk of fatal infection. In addition, despite attempts to increase the donor pool (both by increasing public awareness and by expanding the criteria for donor organ acceptability), the critical and permanent shortage of donor allograft hearts remains a major impediment to the more widespread use of heart transplantation, to the detriment of patients for whom this represents the best potential option.

Future progress will depend on continuing advances in transplantation immunology to minimize the aforemen- 
tioned complications and, most important, in the development of satisfactory alternatives to cardiac allografting. Whether xenografting or mechanical cardiac replacement will be the answer (although these are certainly not mutually exclusive possibilities) largely depends on our (science and society's) commitment to progress in immunology, transplantation, and biomedical engineering.

\section{REFEREN CES}

1. Reitz BA, Bieber CP, Raney AA, et al. Orthotopic heart and combined heart-lung transplantation with cyclosporine A immunosuppression. Transplant Proc 1981;13:393-6.

2. Grattan MT, Moreno-Cabral CE, Starnes VA, Oyer PE, Stinson EB, Shumway NE. Eight-year results of cyclosporine-treated patients with cardiac transplants. J THORAC CARdiovasc Surg 1990;99:500-9.

3. Baumgartner WA, Reitz BA, Oyer PE, Stinson EB, Shumway NE. Cardiac homotransplantation. Curr Probl Surg 1979;16:1-61.

4. Baumgartner WA, Reitz BA, Bieber CP, Oyer PE, Shumway NE, Stinson EB. Current expectations in cardiac transplantation. J THORAC CARdIOvaSC SURG 1978;75: 525-30.

5. Lower RR, Shumway NE. Studies on orthotopic transplantation of the canine heart. Surg Forum 1960;11:1820.

6. Billingham ME, Cary NRB, Hammond ME, et al. International Society for Heart Transplantation: A working formulation for the standardization of nomenclature in the diagnosis of heart and lung rejection: heart rejection study group. J Heart Lung Transplant 1990;9:587-93.

7. Cutler SJ, Ederer F. Maximum utilization of the life table method in analyzing survival. J Chronic Dis 1958;8:699712.

8. SPSS Inc. SPSS-X user's guide. 3d ed. Chicago: 1988.

9. Kaye MP. The Registry of the International Society of Heart and Lung Transplantation: ninth official report1992. J Heart Lung Transplant 1992;11:599-606.

10. Griepp RB, Stinson EB, Dong E Jr, Clark DA, Shumway NE. Determinants of operative risk in human heart transplantation. Am J Surg 1971;122:192-7.

11. Kirklin JK, Naftel DC, Kirklin JW, Blackstone EH, White-Williams C, Bourge RC. Pulmonary vascular resistance and the risk of heart transplantation. J Heart Transplant 1988;7:331-6.

12. Kormos RL, Thompson M, Hardesty RL, et al. Utility of preoperative right heart catheterization data as a predictor of survival after heart transplantation. J Heart Transplant 1986;5:391.

13. Carrier M, Emery RW, Riley JE, Levinson MM, Copeland JG. Cardiac transplantation in patients over 50 years of age. J Am Coll Cardiol 1986;8:285-8.

14. Olivari MT, Antolick A, Kaye MP, Jamieson SW, Ring WS. Heart transplantation in elderly patients. J Heart Transplant 1988;7:258-64.

15. Kirklin JK, Naftel DC, Bourge RC, et al. Rejection after cardiac transplantation-a time-related risk factor analysis. Circulation 1992;86(Suppl):II236-41.

16. Starnes VA, Oyer PE, Stinson EB, Dein JR, Shumway NE. Prophylactic OKT3 used as induction therapy for heart transplantation. Circulation 1989;80(Suppl):III79-83.

17. Renlund DG, O'Connell JB, Gilbert-Hammond ME, et al. A prospective comparison of murine monoclonal $\mathrm{CD}-3$ (OKT3) antibody-based and equine antithymocyte globulin-based rejection prophylaxis in cardiac transplantation. Transplantation 1989;47:599-605.

18. Kirklin JK, Bourge RC, White-Williams C, et al. Prophylactic therapy for rejection following cardiac transplantation: a comparison of RATG and OKT3. J THORAC CARDIOVASC SURG 1990;99:716-24.

19. Frist WH, Oyer PE, Baldwin JC, Stinson EB, Shumway NE. HLA compatibility and cardiac transplant recipient survival. Ann Thorac Surg 1987;44:242-6.

20. Jamieson SW, Oyer PE, Baldwin J, Billingham M, Stinson E, Shumway N. Heart transplantation for end-stage ischemic heart disease: the Stanford experience. Heart Transplant 1984;3:224-7.

21. Gao SZ, Schroeder JS, Alderman EL, et al. Clinical and laboratory correlates of accelerated coronary artery disease in the cardiac transplant patient. Circulation 1987;76(Suppl):V56-61.

22. Gao SZ, Schroeder JS, Alderman EL, et al. Prevalence of accelerated coronary artery disease in heart transplant survivors: comparison of cyclosporine and azathioprine regimens. Circulation 1989;80(Suppl):III100-5.

23. Johnson DE, Alderman EL, Schroeder JS, et al. Transplant coronary artery disease: histopathologic correlations with angiographic morphology. J Am Coll Cardiol 1991;17:44957.

24. Dein JR, Oyer PE, Stinson EB, Starnes VA, Shumway NE. Cardiac retransplantation in the cyclosporine era. Ann Thorac Surg 1989;48:350-5.

25. Grattan MT, Moreno-Cabral CE, Starnes VA, Oyer PE, Stinson EB, Shumway NE. Cytomegalovirus infection is associated with cardiac allograft rejection and atherosclerosis. JAMA 1989;261:3561-6.

26. Gao SZ, Alderman EL, Schroeder JS, Hunt SA, Wiederhold V, Stinson EB. Progressive coronary luminal narrowing after cardiac transplantation. Circulation 1990;82(Sup$\mathrm{pl})$ :IV269-75.

27. Schroeder JS, Gao SZ, Alderman EL, et al. A preliminary study of diltiazem in the prevention of coronary artery disease in heart-transplant recipients. N Engl J Med 1993; 328:164-70.

28. Uretsky BF, Murali S, Reddy PS, et al. Development of coronary artery disease in cardiac transplant patients receiving immunosuppressive therapy with cyclosporine and prednisone. Circulation 1987;76:827-34.

\section{Discussion}

Dr. Adnan Cobanoglu (Portland, Ore.). We have a more modest experience in Portland, Oregon. Since December of 1985, we have performed 218 heart transplants. In going 
through your manuscript, I was taken aback by the similarities both in immunosuppressive regimen and the selection of recipients and donors. As in all centers-in yours, others, and our center-over the years the selection criteria have continued to evolve both for recipients and donors, meaning they all have been liberalized. We are operating on much older recipients over the past few years whom we would never have considered in the early 1980s, and we are also accepting much older donors. Our oldest donor is 58 years of age. This would have been inconceivable in the days when we were writing our protocols for donor selection criteria. Then I was looking for a 20 -year-old donor, a young muscular man who had been shot in the head or had a head injury in a motorcycle accident with no bruises on his chest. Those days are gone never to return.

Our operative mortality is $4.8 \%$. This is considering the same definition that you used-death within 1 month of operative hospitalization or during that hospitalization. One-year survival is $89 \%$ and 5 -year survival is $75 \%$.

The immunosuppressive regimen is triple-drug immunosuppression. It has not changed over the past 7 years and the mainstay is cyclosporine, as in your series. Again, as in your series, we have not realized an increased morbidity or mortality related to operating on more status I patients over the years. About $40 \%$ of our patients are status I at the time of transplantation. They are receiving inotropic drugs or support with a ventilator or cardiac mechanical assist devices. The patient attrition mostly occurs before the transplant operation. Once the transplant operation is done, these patients seem to do quite well.

We have not used induction OKT3 therapy. We have reserved OKT3 for patients who have either renal or other organ dysfunction before transplantation in an effort to delay the first episode of rejection. I wonder if some of the early infectious complications that you observed might be related to the induction OKT3 therapy?

In going through your paper, I did not see very many areas in which we were not in agreement. I agree that grät vasculopathy 3 to 5 years down the line is going to be the major limiting factor and will cause late mortality and morbidity. Fortunately, although a lot of patients seem to have luminal irregularities on surveillance coronary angiograms, these do not necessarily translate into clinical significance.

I have a few questions. The first one concerns the high rate of early mortality caused by infection. You thought that the patients might have rejection and were more aggressively immunosuppressed in the first month. However, $49 \%$ of the deaths were due to infection early on. Isn't this a little higher than figures from most other reports in the literature? It certainly is not our experience. Most of our early deaths are due to either acute right ventricular failure or primary graft dysfunction. Infection as a cause of early death in the Portland experience has not been a major problem.

Dr. Sarris. Regarding the infection rate with OKT3, we have looked at infections overall as well as viral infections and CMV infections in particular and have found no difference in the occurrence of these infections before and after OKT3. Now, again, these are not concurrent studies. It is a retrospective analysis, but nonetheless on retrospective review, we have not found OKT3 to be associated with one infection.

The risk of early death seemed to be related in our experience to pulmonary hypertension. That pertains to death in the hospital. I believe our operative mortality is higher than that reported from other centers because the pediatric operative mortality was higher; however, this excess mortality in the pediatric group, which was approximately $16 \%$ to $18 \%$, could be accounted for by the higher prevalence of patients in that group with higher preoperative PVR. The deaths due to infection occur, again, during the first year, and this has just been our experience. I really cannot explain it otherwise.

Dr. Cobanoglu. Has your infectious prophylaxis changed over the years? You said that you did better over the past few years. Have you changed your isolation protocols or antibiotic regimen perioperatively? What do you use now?

Dr. Sarris. Antibiotic prophylaxis has been the same throughout the years. We have used cefamandole prophylaxis perioperatively for 48 hours. We have also used erythromycin because we had some difficulties in our intensive care unit with Legionella colonization with the water supply. This has not changed over the years. I believe we have actually had fewer infections in the more recent years. In fact, as medical economics have put pressure on the practice of medicine, including transplantation, we have relaxed some of the in-hospital precautions in terms of isolating heart transplant patients. They are no longer being isolated. The infections that do occur I just cannot further explain.

Dr. Cobanoglu. How is your experience retransplantation? Our results are not very good.

Dr. Sarris. This study specifically excluded patients who underwent retransplantation. The patients who did undergo retransplantation were included in all the survival data analysis because we were interested in patient rather than graft survival, but they were excluded from analysis of other complications. Approximately 40 patients underwent retransplantation, and 9 of those are currently alive. They have in general done worse than patients receiving primary transplants. 